\title{
Getting Routine Intimate Partner Violence Screening Right: Implementation Strategies Used in Veterans Health Administration (VHA) Primary Care
}

\author{
Omonyêté L. Adjognon, ScM, Julianne E. Brady, MA, Megan R. Gerber, MD, MPH, \\ Melissa E. Dichter, PhD, MSW, Alessandra R. Grillo, BS, Alison B. Hamilton, PhD, MPH, \\ Shannon Wiltsey Stirman, PhD, and Katherine M. Iverson, PhD
}

Background: Evidence supports the clinical effectiveness of intimate partner violence (IPV) screening programs, but less is known about implementing and sustaining them. This qualitative study identified implementation strategies used to integrate IPV screening programs within Veterans Health Administration (VHA) women's health primary care.

Methods: Thirty-two administrators and clinician key informants from 11 VHA facilities participated in semistructured interviews. Implementation strategies were identified using established definitions from implementation science literature, through multistep content analysis, involving site comparisons by implementation status.

Results: We identified 8 implementation strategies. Three were present across all sites: (1) conduct ongoing IPV trainings, (2) conduct educational meetings and outreach visits, and (3) develop and distribute educational materials. Five strategies were unique to early adopting sites: (4) identify and prepare champions, (5) change record systems to remind clinicians, (6) create a learning collaborative through advisory boards or workgroups, (7) audit and provide feedback with relay of clinical data to providers, and (8) access new funding.

Discussion: Strategies align with and extend literature addressing barriers to screening. Evidence shows that effective IPV screening implementation in primary care requires a bundle of well-defined, carefully selected strategies.

Conclusions: Implementation strategies used collectively can enable integration of IPV screening programs in primary care. ( $\mathrm{J}$ Am Board Fam Med 2021;34:346-356.)

Keywords: Implementation Science, Intimate Partner Violence, Primary Health Care, Qualitative Research, Veterans Health Administration, Women's Health

Intimate partner violence (IPV) is a substantial threat to women's safety and health. ${ }^{1}$ Although individuals of any gender identity can experience

This article was externally peer reviewed.

Submitted 24 June 2020; revised 4 September 2020; accepted 20 September 2020.

From the Women's Health Sciences Division, National Center for PTSD, VA Boston Healthcare System, Boston, MA (OLA, JEB, ARG, KMI); Center for Healthcare Organization and Implementation Research (CHOIR), VA Boston Healthcare System, Boston, MA (OLA, JEB); Women's Health Center, VA Boston Healthcare System, Boston MA (MRG); Section of General Internal Medicine, Boston University School of Medicine, Boston, MA (MRG); VA Center for Health Equity Research and Promotion (CHERP), Crescenz VA Medical Center, Philadelphia, PA (MED); School of Social Work, Temple University, Philadelphia, PA (MED); Department of Psychology, University of North Carolina at Greensboro, Greensboro, NC (ARG); VA Center for the Study of Healthcare Innovation,
IPV, women are disproportionately affected, with elevated prevalence, severity, and health impacts. ${ }^{1}$ Within the United States, at least 1 in 4 women

Implementation and Policy (CSHIIP), VA Greater Los Angeles Healthcare System, Los Angeles, CA (ABH); Department of Psychiatry and Biobehavioral Sciences, University of California Los Angeles David Geffen School of Medicine, Los Angeles, CA (ABH); Dissemination and Training Division, National Center for PTSD, VA Palo Alto Healthcare System, Palo Alto, CA (SWS); Department of Psychiatry and Behavioral Sciences, Stanford University School of Medicine, Stanford, CA (SWS); Department of Psychiatry, Boston University School of Medicine, Boston MA (KMI).

Funding: This research was supported by the Department of Veterans Affairs, Veterans Health Administration, Health Services Research and Development (HSR\&D) Services (PPO 17-044; PI Iverson). This work was also supported, in part, by KMI's Presidential Early Career Award for Scientists 
experience physical violence, sexual violence, or stalking by a partner that results in health-related impacts (eg, fear, safety concerns, injury, psychological symptoms, and need for medical care). ${ }^{2}$ This troubling prevalence estimate does not include psychological IPV, which has particularly adverse effects on health. ${ }^{3,4}$ IPV is associated with higher health care use, including higher primary care use. ${ }^{5,6}$ Supported by evidence from a systematic review, ${ }^{7}$ the US Preventive Services Task Force recommends IPV screening, counseling, and offering referrals during primary care visits for women, ${ }^{8}$ a cluster of practices referred to as "IPV screening programs." 9 Such programs enable primary care providers (PCPs) to offer emotional and tangible support, resources, and referrals to services that can enhance safety and health. ${ }^{10}$ But implementing any new clinical practice or changes to clinical practice is challenging, particularly for a complex, stigmatized public health problem like IPV. ${ }^{11}$

Despite the proliferation of IPV curriculum, ${ }^{12}$ few states in the United States currently require IPV screening continuing medical education, ${ }^{13}$ and the level of PCP knowledge about IPV identification and referral for patients who endorse IPV is modest and highly variable. In fact, only $2 \%$ to $50 \%$ of all health care providers report screening for IPV on a regular basis. ${ }^{14}$ With limited provider education and personal discomfort reported as major barriers to screening, ${ }^{13,15-18}$ IPV training for PCPs is critical. Provider educational efforts are important implementation strategies in response to this gap; however, training alone is not sufficient for sustained adoption. Provider education is associated with short-term increases in provider screening rates, but studies suggest its insufficiency, as provider-education-focused implementation efforts show no significant long-term effect on screening uptake or identification rates over time. ${ }^{19}$

and Engineers (USA 14-275) and Implementation Research Institute fellowship from the National Institute of Mental Health (5R25MH08091607) through HSR\&D Services, Quality Enhancement Research Initiative.

Conflict of interests: None declared.

Disclaimer: The views expressed in this article are those of the authors and do not necessarily reflect the position or policy of the Department of Veterans Affairs or the United States government.

Corresponding author: Omonyêlé Adjognon, ScM, Women's Health Sciences Division, National Center for PTSD, VA Boston Healthcare System, 150 South Huntington Avenue (116B-3), Boston, MA 02130 (E-mail: omonyele.adjognon@va.gov).
In addition, there are common logistical barriers such as time constraints, competing priorities within visits, and lack of referral options for counseling or support services. ${ }^{13,16,18,20}$ Additional implementation strategies used to promote IPV screening include (1) giving providers screening guidelines or protocols, ${ }^{19}$ (2) placing screening prompts for providers in patients' health records ${ }^{21}$ or changing the record system to provide reminders in patients' health records, ${ }^{2-25}$ (3) having a designated victim advocate onsite, ${ }^{19}$ (4) involving executive boards ${ }^{24}$ or stakeholder groups, ${ }^{26}$ and (5) soliciting feedback from staff and other clinic representatives. ${ }^{26,27}$ However, it is unclear from the literature how effective each of these strategies is on their own or when used in combination. Thus, it remains unknown what package of implementation strategies is needed for effective and sustainable implementation of IPV screening programs.

The integration of implementation science into IPV screening research and evaluation can help close this gap. ${ }^{28-30}$ Within the implementation science literature, the Expert Recommendations for Implementing Change (ERIC) project ${ }^{31}$ established a set of 73 clearly conceptualized implementation strategies to guide consistent definitions in research and practice. Although ERIC has been used for understanding the implementation of other health care interventions (eg, see Perry et $\mathrm{al}^{32}$ ), there are no published studies assessing strategies for successful IPV screening implementation purposefully using the ERIC compilation. There remains a need to identify key implementation strategies for integrating IPV screening programs in primary care, using conceptually clear and consistent definitions. ${ }^{33}$

IPV is a critical issue for the Veterans Health Administration (VHA), ${ }^{34}$ the largest integrated health care system in the nation, as nearly 1 in 5 (18.5\%) women using VHA primary care have experienced past-year IPV. ${ }^{6}$ As VHA started implementing IPV screening programs in primary care in 2014, as recommended by the VA Domestic Violence/IPV Task Force, ${ }^{35}$ there is an opportunity to learn about implementation strategies used in this context. Specifically, the use of discrete implementation strategies across VHA Medical Centers (VAMCs) in attempts to integrate IPV screening programs remains unknown.

This study uses the ERIC compilation of implementation strategies ${ }^{31}$ to identify discrete implementation strategies used at VAMCs to implement IPV screening programs in primary care clinics 
across the country, through a qualitative study with VHA health care providers and administrators. Unlike prior research that mostly looks at controlled IPV screening trials and interventional studies, ${ }^{9}$ the current study focuses on naturally occurring strategies used in VHA. Our goal is to document implementation strategies collectively used for implementation of routine IPV screening across VHA primary care clinics. Findings can inform the spread of IPV screening programs across VHA as well as implementation strategies for other integrated health care systems.

\section{Method}

\section{Participants}

With study approval from the Institutional Review Board of the VA Boston Healthcare System, we recruited 11 VAMCs nationwide, at varying stages of IPV screening program adoption in primary care. From these sites, we enrolled and interviewed 32 key informant clinicians and administrators with knowledge of or involvement with IPV screening and response programming in their local women's health primary care clinics (eg, women's health medical director, PCP, primary care social worker, IPV Assistance Program coordinator, women veterans program manager). Additional details regarding participants and recruitment procedures are published elsewhere. ${ }^{20}$

\section{Procedures}

Sites were selected using purposive sampling ${ }^{36}$ based on program evaluation findings from our operational partners in VHA's Office of Women's Health Services. Women's health primary care staff completed brief surveys regarding current adoption status. Early adopting sites were defined as those that reported currently engaging in IPV screening as part of routine care (ie, screening all female patients of childbearing age or all female patients at least annually). Late-adopting sites were defined as those that reported not currently engaging in IPV screening as routine care (ie, screening at provider's discretion). ${ }^{20}$ We recruited and enrolled 6 early adopting sites and 5 late-adopting sites for this study. Sites were geographically spread across the United States.

The research team, which included experts in IPV, trauma-informed primary care, qualitative research, and implementation science, developed a semistructured interview guide based on the integrated-Promoting Action on Research Implementation in Health Services (i-PARIHS) framework. ${ }^{37}$ i-PARIHS provided a conceptual framework to characterize the ways in which IPV screening practices and implementation strategies have been used in VHA. Questions focused on understanding the history of IPV screening program implementation efforts at the site. Sample questions included: "How was IPV screening initially started at your primary care clinic(s) and who was involved in that?", "What helped you to implement IPV screening or what has made it easier?", and "What have been some of the challenges and what did your facility do to overcome them?" Questions were designed to be flexible, and the interviewer asked follow-up questions and probed for examples to understand strategies used to support implementation.

\section{Data Collection}

Between October 2017 and February 2018, the last author conducted all one-on-one, semistructured phone interviews with 32 key informants across 11 sites. As much as possible, participants were asked the same questions (using the interview guide) regardless of their site's IPV screening adoption status. Interviews were recorded with participants' permission and transcribed verbatim.

\section{Data Analysis}

Rapid content analysis ${ }^{38}$ was applied to identify implementation strategies used at study sites. We created a codebook based on the refined compilation of implementation strategies from the ERIC project. ${ }^{31}$ This compilation provides a comprehensive list of implementation strategies that can be used in isolation or combination to support implementation efforts, ${ }^{31}$ our team selected 20 strategies a priori most relevant to IPV screening program implementation and adapted their definition accordingly (eg, replacing "clinical innovation" by "IPV screening"). The code "other implementation strategies" was also added to inductively capture strategies beyond those identified deductively, resulting in a 21-item codebook used for analysis. Four team members trained in qualitative approaches then independently coded 4 transcripts using the deductive codebook, convened to discuss and refine codebook definitions, and reached consensus across codes and transcripts. ${ }^{38}$ Thereafter, each of the 28 remaining transcripts was coded by 2 
Figure 1. Qualitative data analysis steps.

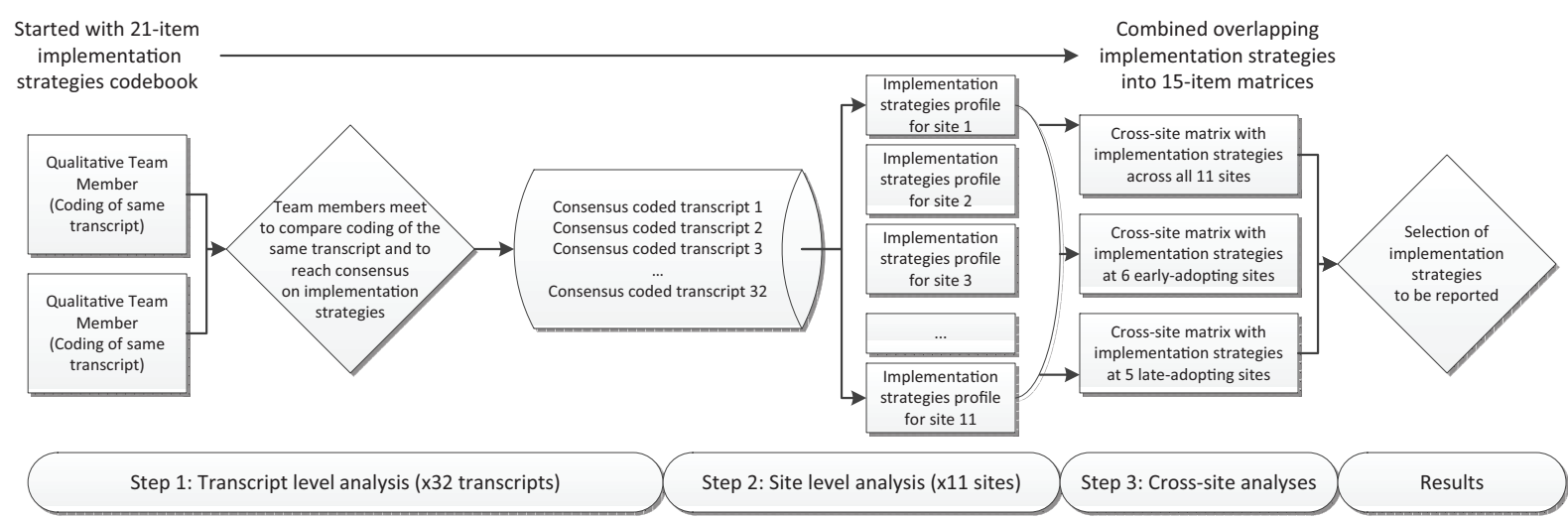

team members with ongoing consensus meetings. Data analysis steps are summarized in Figure 1.

\section{Step 1: Transcript-Level Analysis}

Our team reviewed each transcript against the 21item codebook, selecting illustrative quotes for each identified strategy. A strategy could receive multiple codes. Each transcript was coded by 2 team members separately, who then met routinely for an hour on average and discussed discrepancies to reach consensus.

\section{Step 2: Site-Level Analysis}

As each site had 2 to 4 participants (32 participants across 11 sites), consensus codes from transcripts were merged to create each site's implementation strategies profile on an Excel spreadsheet. Each row represented a strategy, while 3 columns contained the codebook definition, summary evidence, and illustrative quotes for that site.

\section{Step 3: Cross-Site Analysis}

We combined site profiles into a cross-site Excel matrix where 21 rows listed all implementation strategies codes, and 11 columns summarized each site's evidence per strategy. On cross-site matrix review, an empty row labeled "provide clinical supervision" was removed due to lack of evidence at any site. In performing matrix content analysis, we observed overlap of evidence across several strategies, consistent with the interconnectedness found between ERIC-based strategies in prior screening trials. ${ }^{32}$ Hence, we combined 5 of the 20 rows with the remaining 15 , and tailored strategy definitions and evidence accordingly. For example, "audit and provide feedback" was combined with "facilitate relay of clinical data to providers," because the team agreed that available evidence for both strategies was the same per their respective definitions. Similarly, overlaps of evidence between other categories, including: (1) "conduct educational meetings" and "conduct educational outreach visits"; (2) "change record systems" and "remind clinicians" to screen for IPV; (3) "create a learning collaborative" and "use advisory board or workgroups"; and (4) "develop IPV education materials" and "distribute IPV educational materials" led to additional categorical merging.

The final cross-site matrix from which findings were derived had 15 refined implementation strategies across all sites. We created 2 submatrices to examine strategies across early adopting and lateadopting sites, separately.

\section{Results}

Our analysis highlighted 8 implementation strategies, each evidenced by 3 or more sites in our 11site sample. These strategies and definitions are displayed in Table 1 . We categorized them into 2 groups: (1) strategies present across sites regardless of IPV screening program adoption status, and (2) strategies present only in early adopting sites. We found no strategies unique to late-adopting sites. Table 2 displays exemplar quotes for all 8 implementation strategies. It is also notable that while early adopting sites each used on average 4.25 of these 8 implementation strategies, late-adopting sites engaged with 2.67 such strategies on average (see Table 3). 
Table 1. Definitions of Intimate Partner Violence Screening Implementation Strategies Used

\begin{tabular}{|c|c|}
\hline Implementation Strategies & Definitions \\
\hline Access new funding & Access new or existing money to facilitate IPV screening implementation \\
\hline $\begin{array}{l}\text { Audit and provide feedback, with relay of } \\
\text { clinical data to providers }\end{array}$ & $\begin{array}{l}\text { Collect and summarize clinical performance data over a specified time period about } \\
\text { key measures of process/outcomes, then give it to clinicians and administrators to } \\
\text { modify provider behavior and promote use of IPV screening practices }\end{array}$ \\
\hline Change record systems to remind clinicians & $\begin{array}{l}\text { Change record systems to allow better assessment of implementation or clinical } \\
\text { outcomes (eg, clinical reminder, note templates) and to prompt clinicians to use } \\
\text { the IPV screening clinical innovation }\end{array}$ \\
\hline $\begin{array}{l}\text { Conduct IPV educational meetings and } \\
\text { educational outreach visits }\end{array}$ & $\begin{array}{l}\text { Hold meetings led by a trained person and targeted toward different stakeholder } \\
\text { groups (eg, providers, administrators, other organizational stakeholders, and } \\
\text { community, patient/consumer, and family stakeholders) to teach them about IPV } \\
\text { and IPV screening }\end{array}$ \\
\hline Conduct ongoing IPV trainings & Plan for and conduct IPV trainings in an ongoing way \\
\hline $\begin{array}{l}\text { Create a learning collaborative through } \\
\text { advisory boards or workgroups }\end{array}$ & $\begin{array}{l}\text { Facilitate the formation of groups of providers and other kinds of stakeholders' } \\
\text { groups that foster a collaborative learning environment, to provide input and } \\
\text { advice on implementation efforts and improve IPV screening implementation }\end{array}$ \\
\hline $\begin{array}{l}\text { Develop and distribute IPV educational } \\
\text { materials }\end{array}$ & $\begin{array}{l}\text { Develop and format IPV guidelines, manuals, toolkits, and other supporting } \\
\text { materials in ways that make it easier for stakeholders to learn about IPV, then } \\
\text { distribute these materials in person, by mail, and/or electronically to enable staff } \\
\text { and clinicians to learn how to deliver IPV screening }\end{array}$ \\
\hline Identify and prepare champions & $\begin{array}{l}\text { Identify and prepare individuals who dedicate themselves to supporting, marketing, } \\
\text { and driving through IPV screening implementation, overcoming indifference or } \\
\text { resistance that the intervention may provoke within the medical center }\end{array}$ \\
\hline
\end{tabular}

IPV, intimate partner violence.

Adapted from Powell et al. ${ }^{31}$

\section{Implementation Strategies across All Sites}

Three strategies were common across early and late-adopting sites.

\section{Conduct Ongoing IPV Trainings}

Ongoing trainings that built on each other over time enabled providers (eg, physicians, nurses, social workers) to effectively integrate IPV screening practices. Indepth trainings were various combinations of basic IPV education: tips on talking about IPV with patients using trauma-informed care principles; roleplays to help providers gain firsthand experience with screening and responding and problem-solving potentially challenging situations; clarifying clinical pathways following disclosures; how to offer education, validation, and support; and resource options. Trainings were presented in different formats: regular dedicated training events, trainings incorporated into larger staff events, retraining where deficiencies in screening delivery were identified, and additional one-on-one trainings to fill gaps outside of scheduled training events.

\section{Conduct Educational Meetings and Educational Outreach Visits}

Sites discussed intermittent "bite-sized" IPV screening education delivered to other relevant providers and stakeholders. IPV-trained staff (eg, psychiatrist, social worker) used events such as town hall meetings or discipline-specific events (eg, primary care leadership meetings) to deliver IPV screening education in various formats (ie, lectures, case examples, and art projects). These efforts included traveling to external locations such as community-based outpatient clinics in both rural and urban settings to share this knowledge. Educational information included IPV statistics and context to enhance provider knowledge and buy-in. Educational meetings and educational outreach visits differed from ongoing IPV trainings. Such meetings were not ongoing. They focused on the delivery of easily grasped information to increase awareness of IPV and screening recommendations. In contrast, conducting ongoing IPV trainings speaks to the repetitive, continuous nature of indepth knowledge and skill transfer to improve clinical practice.

\section{Develop and Distribute IPV Educational Materials} Sites either created or adapted materials used for staff and providers' IPV training (eg, presentations, handouts); compiled, maintained, and shared lists of community resources on IPV with providers; and 
Table 2. Intimate Partner Violence Screening Implementation Strategies with Exemplar Quotes for Strategies Across Early and Late-Adopting Sites, and Early Adopting Sites Only

Implementation Strategies

Exemplar Quotes

Implementation Strategies across Early and Late-Adopting Sites Conduct ongoing IPV trainings

Conduct educational meetings and educational outreach visits

Develop and distribute IPV educational materials

Implementation Strategies across Early Adopting Sites Only Identify and prepare champions

Change record systems to remind clinicians

Create a learning collaborative through advisory boards or workgroups

"I thought that the primary care social worker could have used a little more training early on, so I sat down with her and did some more training." -IPV Assistance Program Coordinator, early adopting site

"All the consistent training I've been doing for the past 1.5 years is working because now the calls I'm getting from providers are consultation calls and not calls of 'I don't know what to do with this, I don't know what the resources are, can you help them, can you fix it?' It's more of, 'I did this, this, and this and now, I'm still concerned about how we can help her with $\mathrm{x}, \mathrm{y}$, and $\mathrm{z}$."' -IPV Assistance Program Coordinator, late-adopting site

"I present whenever I can, so I present at our town hall meetings, I present at the nurses/RN awareness week, and then social work week. I really try to be as active as I can with supervisors, and then going down the line in the actual clinic." -IPV Assistance Program Coordinator, early adopting site

"We have focused on staff education where I've gone to and given presentations on this to the social work line, to our mental health line, to mental health nurses, etc as much as possible within my own clinic, and so we focused on staff education." -IPV Assistance Program Coordinator, lateadopting site

"One of the things that the IPV Coordinator did do when she first came on was she put together a resource list, both for the two counties that the two medical centers are in, and also through all the counties that the 7 community-based outpatient clinics are in... and I think she sent it out to everybody." -Women Veterans Program Manager, lateadopting site

"We have handouts and posters in the waiting rooms, in the clinic, at our community-based outpatient clinics... we've really made an effort to spread the word in the VA system." Women's Health Medical Director and Primary Care Physician, early adopting site

"[IPV Coordinator] is a powerhouse, and she has been able to make things happen that nobody else before her was able to do... she was such a work horse that we all kind of looked up, and there it was. She's the one that put this into place and made it happen. She gave us the ability to train the people with whom we work. IPV screening necessitates a warm handoff to a social worker if it's positive, so getting everyone trained and the ability to train others has been an important part of putting it into place." -Primary Care Social Worker

"It initially got started as a clinical reminder that the licensed practice nurse would complete during their intake phase, and then if it screened positive, it would go to the provider to do more assessment." -Primary Care Physician

"I got people from primary care, I got the chaplain involved, I got the police department involved, I got a nurse from the [emergency department], social work got involved, psychiatry got involved, [human resources] got involved,... and it's 1 hour bimonthly and it's very planned so they get an agenda about what we'll be talking about and we stick pretty firm to that and I ask how they might be available to help. . .that's actually how I got the pledge screening signed." -IPV Assistance Program Coordinator 


\begin{tabular}{|c|c|}
\hline Implementation Strategies & Exemplar Quotes \\
\hline $\begin{array}{l}\text { Audit and provide feedback, with relay of clinical data to } \\
\text { providers }\end{array}$ & $\begin{array}{l}\text { "That was something that I started sending out to clinics, } \\
\text { saying, 'This is where your clinic is at, this is where it's not.' } \\
\text { Because I think numbers speak more than what I can, and so } \\
\text { when I came on board, we were only at } 32 \% \text { hospital wide. } \\
\text { And I have received them every month now for a little over a } \\
\text { year, and they went from } 32 \% \text { to } 77 \% \text {." -IPV Assistance } \\
\text { Program Coordinator }\end{array}$ \\
\hline Access new funding & $\begin{array}{l}\text { "Then she applied for the second grant, and with the second } \\
\text { grant, it was the implementation of the actual clinical } \\
\text { reminder, the computer version of the recommended } \\
\text { screening tool." -Women Veterans Program Manager }\end{array}$ \\
\hline
\end{tabular}

IPV, intimate partner violence.

developed screening protocols and documentation guidelines for teams to use when screening patients for IPV. Materials were distributed in the facility either electronically through e-mail and SharePoint (eg, PowerPoint slidedeck that departments can tailor to fit their needs), via flyers posted in common areas, or in person (eg, training sessions, PCPs giving patients brief handouts or brochures).

\section{Implementation Strategies across Early Adopting Sites Only}

Five implementation strategies were present only in early adopting sites and are described next.

\section{Identify and Prepare Champions}

For all early adopting sites, having dedicated IPV champions was essential to implementation. Champions' dedication to driving IPV screening implementation manifested through: (1) regular participation in national calls/meetings led by VHA leadership to access a wider network of resources; (2) relationship building with the community (ie, meetings, summits) and gaining access to community networks and resources; (3) training and educating staff and providers; (4) champions in leadership promoting a clinical reminder to facilitate screening; and (5) champions conducting IPV screening embedded within the clinic for efficiency, tailoring the process to local contextual needs.

\section{Change Record Systems to Remind Clinicians}

Most sites used electronic medical records systems to remind clinicians, thus facilitating adoption of, and comfort with, IPV screening practices. Specifically: (1) clinical reminders prompted routine IPV screening and response practices; (2) screening electronically made it easier for PCPs to record screening results, additional assessments, and clinical resources provided; (3) the ability to turn on the clinical reminder for only some PCPs made piloting and refining IPV screening protocols possible without having to train all hospital PCPs beforehand; and (4) tracking positive screens created useful data to refine the screening process.

\section{Create a Learning Collaborative through Advisory Boards or Workgroups}

Most sites created groups of stakeholders involved in IPV screening program implementation, giving/ receiving support and advice, and sharing knowledge. Some local workgroups (ie, advisory board, committee, taskforce) had stakeholders from different areas (ie, IPV experts, medical university staff, social workers, veterans). They met regularly to coordinate IPV screening-related tasks (ie, educational events), develop relationships with relevant areas of the hospital, and problem-solve challenges. In addition, they called in external experts from the community to aid in assessment of IPV and community referrals. Sites that were further along played a mentoring role to sites in the process of implementation. Lastly, access to a broader network provided opportunities for more informal connections and collaborations, serving both to implement and, when relevant, sustain IPV screening programs.

Audit and Provide Feedback, with Relay of Clinical Data to Providers

Most sites collected and analyzed data on their process to promote IPV screening uptake and optimization, all the while informing PCPs on program 
Table 3. Implementation Strategies Across Sites by Intimate Partner Violence Screening Adoption Status

\begin{tabular}{|c|c|c|c|}
\hline \multirow[b]{2}{*}{ Implementation Strategies } & \multirow[b]{2}{*}{ All Sites $(n=11)$} & \multicolumn{2}{|c|}{ Variation by IPV Screening Adoption Status } \\
\hline & & Early Adopting Sites $(\mathrm{n}=6)$ & Late-Adopting Sites $(\mathrm{n}=5)$ \\
\hline Conduct ongoing IPV trainings & 8 sites & 6 sites & 2 sites \\
\hline $\begin{array}{l}\text { Conduct educational meetings } \\
\text { and outreach visits* }\end{array}$ & 6 sites & 4 sites & 2 sites \\
\hline $\begin{array}{l}\text { Develop and distribute IPV } \\
\text { educational materials* }\end{array}$ & 8 sites & 4 sites & 4 sites \\
\hline Identify and prepare champions & 6 sites & 6 sites & None \\
\hline $\begin{array}{l}\text { Change record systems to } \\
\text { remind clinicians* }\end{array}$ & 5 sites & 5 sites & None \\
\hline $\begin{array}{l}\text { Create a learning collaborative } \\
\text { through advisory board or } \\
\text { workgroups }\end{array}$ & 5 sites & 5 sites & None \\
\hline $\begin{array}{l}\text { Audit and provide feedback, with } \\
\text { relay of clinical data to } \\
\text { providers }\end{array}$ & 4 sites & 4 sites & None \\
\hline Access new funding & 3 sites & 3 sites & None \\
\hline
\end{tabular}

IPV, intimate partner violence.

*Some late-adopting sites were not included because they mentioned these implementation strategies as plans but not actual actions yet.

Mean implementation strategies used per site:

Across all sites, 5.62 implementation strategies.

For early adopting sites, 4.25 implementation strategies.

For late-adopting sites, 2.67 implementation strategies.

implementation progress and areas for growth. Concretely, these data were used to demonstrate the need for an IPV Assistance Program coordinator or a clinical reminder, show screening deficiencies, assess IPV screening success, and determine next steps. Sites already using electronic clinical reminders for IPV screening could more easily monitor and share program performance.

\section{Access New Funding}

A few sites secured new or existing money from VHA leadership to facilitate the implementation of IPV screening. Funding included grants to create a clinical reminder and distribute resources to patients who endorsed IPV and a dedicated IPV Assistance Program coordinator position to protect time and resources for supporting IPV screening program implementation and sustainment.

\section{Discussion}

Evidence supports the clinical effectiveness of IPV screening programs, but implementation in realworld care is challenging. This study identifies 8 implementation strategies used to support IPV screening program implementation in the context of VHA primary care.

It is not surprising that 3 of these strategies, evidenced across early and late-adopting sites (ie, conduct ongoing IPV trainings, conduct educational meetings and educational outreach visits, and develop and distribute IPV educational materials), focus on training and education with adequate supporting materials. This is consistent with literature identifying provider education and personal discomfort with IPV as primary barriers to IPV screening. ${ }^{14,19}$ Identified implementation strategies pertaining to developing and distributing educational materials (eg, sharing a list of community resources with providers to enable tailored referrals) also align with prior literature indicating that adding clinical tools and guidelines (eg, screening protocols, referral lists) to education efforts tends to improve the implementation and clinical effectiveness of IPV screening programs. ${ }^{9}$ These foundational implementation strategies may be relatively more feasible and lower cost compared with other more intensive implementation strategies. Regardless, these 3 strategies seem to be a primer for all sites in propelling screening forward. 
We identified 5 additional implementation strategies being used uniquely in early adopting sites. These strategies were likely selected to overcome specific barriers confronted during the early stages of implementation at these sites. These additional strategies align well with common barriers to IPV screening identified in prior research. They confirm the need for additional implementation strategies (beyond training and education) in addressing barriers to implementation; namely, (1) identifying and preparing champions, including social work and mental health providers with specific expertise in IPV and who can help identify clinical pathways following disclosures; ${ }^{20,39}$ (2) auditing and providing feedback with relay of clinical data to providers; and (3) accessing new funding, collectively heightening the perceived importance of IPV screening programs. $^{20,40}$ In addition, (4) changing record systems to remind clinicians helps mitigate time constraint; ${ }^{13,16,18,20}$ and (5) creating a learning collaborative through advisory boards or workgroups ensures widespread understanding of the impact of IPV on women's health. ${ }^{13,30}$ These 5 strategies are also concordant with strategies used to complement providers' IPV education as noted in prior literature (eg, having an onsite clinician to provide follow-up services, involving executive board or stakeholder groups, and eliciting staff feedback). ${ }^{21-}$ 24,26,27 Although late-adopting sites had not used these strategies at the time of this study's data collection, it is possible that these sites eventually used one or more of these strategies as their implementation efforts continued. Additional site-specific barriers likely contributed to the selection of strategies at different sites.

The fact that early adopting sites used these 8 implementation strategies (average 4.25 strategies per early adopting site compared with 2.67 strategies per late-adopting site) suggests that multiple implementation strategies used in tandem facilitate routine IPV screening and response practices. It is also likely that contextual factors, such as resources and number of providers in the clinic, contributed to successful adoption. Indeed, there were indicators that early adopting sites had greater staffing and capacity (eg, IPV champion in place and grant funding) than late-adopting ones, which may have contributed to greater on-site IPV screening expertise.

This study has identified strategies used collectively to implement IPV screening programs in
VHA primary care. Strengths include the use of an established implementation science framework and methods. The use of ERIC to identify implementation strategies extends the IPV screening literature and was feasible with tailoring of the definitions to our study. This included (1) allowing multiple codes for a strategy when appropriate, (2) using a manageable number of codes (eg, selecting 20 out of 73 strategies available), and (3) combining overlapping codes at the analysis stage. These findings could inform the establishment and testing of a comprehensive toolkit of implementation strategies for IPV screening implementation across VHA primary care settings, to which the present 8 strategies are a start.

This study has several limitations. This study was conducted within VHA, and it is possible that these strategies may not be adequate in other settings, especially in nonintegrated care contexts or those without IPV clinical experts. The IPV Assistance Program coordinators are fairly unique to VHA, and they were often the most knowledgeable about implementation efforts. Findings may not translate well to smaller health care systems and practices with a narrower scope that do not have the availability of an on-site IPV consultant. However, the expansion of mental/behavioral health services within primary care clinics offers opportunities for enhancing IPV detection and care. Another limitation is that other implementation strategies not subsumed within our selected a priori codes from the ERIC compilation would not be captured in this study. Therefore, it is possible that other important implementation strategies used across early and late-adopting sites were not identified. However, strategies identified under "other implementation strategies" were minimal, signaling this as a minor limitation. Future studies could use surveys to assess for ERIC-based implementation strategy use in IPV screening implementation efforts.

Successful integration of IPV screening programs within routine care is critical in addressing the public health burden of IPV. Understanding promising implementation strategies used in previous IPV screening efforts in VHA is timely. As of January 2019, VHA policy requires IPV screening as a standard of care. ${ }^{41}$ VHA has already initiated a larger-scale effort to spread IPV screening programs nationwide using the strategies highlighted in this study with a complementary mixed-method evaluation of the implementation impact. ${ }^{30}$ Resulting knowledge will further guide effective 
IPV screening implementation in health care, beyond VHA primary care. Done right, routine IPV screening can significantly reduce IPV-related adverse outcomes to women's health.

Our special thanks to Rachel M. Maskin for her assistance with data analysis, Cassidy Gutner, $\mathrm{PhD}$, for her comments on the original study design, and to the clinicians and administrators who shared their perspectives for this research. We also thank VHA's Office of Women's Health Services and the VHA IPV Assistance Program of Care Management and Social Work Services for providing program evaluation metrics for sampling and recruitment.

To see this article online, please go to: http://jabfm.org/content/ 34/2/346.full.

\section{References}

1. World Health Organization. Responding to intimate partner violence and sexual violence against women: WHO clinical and policy guidelines. Geneva, Switzerland: World Health Organization; 2013.

2. Smith SG, Zhang X, Basile KC, et al. The national intimate partner and sexual violence survey: 2015 data brief-updated release. 2018.

3. Coker AL, Davis KE, Arias I, et al. Physical and mental health effects of intimate partner violence for men and women. Am J Prev Med 2002;23:260-8.

4. Dichter ME, Sorrentino A, Bellamy S, Medvedeva E, Roberts CB, Iverson KM. Disproportionate mental health burden associated with past-year intimate partner violence among women receiving care in the Veterans Health Administration. J Trauma Stress 2017;30:555-63.

5. Bonomi AE, Anderson ML, Reid RJ, Rivara FP, Carrell D, Thompson RS. Medical and psychosocial diagnoses in women with a history of intimate partner violence. Arch Intern Med 2009;169:1692-7.

6. Kimerling R, Iverson KM, Dichter ME, Rodriguez AL, Wong A, Pavao J. Prevalence of intimate partner violence among women veterans who utilize Veterans Health Administration primary care. J Gen Intern Med 2016;31:888-94.

7. Feltner C, Wallace I, Berkman N, et al. Screening for intimate partner violence, elder abuse, and abuse of vulnerable adults: evidence report and systematic review for the US Preventive Services Task Force. JAMA 2018;320:1688-701.

8. US Preventive Services Task Force. Screening for intimate partner violence, elder abuse, and abuse of vulnerable adults: US Preventive Services Task Force final recommendation statement. JAMA 2018;320:1678-87.

9. O'Campo P, Kirst M, Tsamis C, Chambers C, Ahmad F. Implementing successful intimate partner violence screening programs in health care settings: evidence generated from a realist-informed systematic review. Soc Sci Med 2011;72:855-66.

10. Miller E, McCaw B. Intimate partner violence. N Engl J Med 2019;380:850-7.

11. Young-Wolff KC, Kotz K, McCaw B. Transforming the health care response to intimate partner violence: addressing “wicked problems.” JAMA 2016;315:2517-8.

12. Association of American Medical Colleges. Content documentation in required courses and elective courses: topic of domestic violence/abuse. Available from: https://www. aamc.org/data-reports/curriculum-reports/interactivedata/content-documentation-required-courses-andelective-courses. Updated 2020. Accessed June 17, 2020.

13. Phares TM, Sherin K, Harrison SL, Mitchell C, Freeman R, Lichtenberg K. Intimate partner violence screening and intervention: the American College of Preventive Medicine position statement. Am J Prev Med 2019;57:862-72.

14. Alvarez C, Fedock G, Grace KT, Campbell J. Provider screening and counseling for intimate partner violence: a systematic review of practices and influencing factors. Trauma Violence Abuse 2017;18:479-95.

15. Gotlib Conn L, Young A, Rotstein OD, Schemitsch E. "I've never asked one question." Understanding the barriers among orthopedic surgery residents to screening female patients for intimate partner violence. Can J Surg 2014;57:371-8.

16. Sprague S, Madden K, Simunovic N, et al. Barriers to screening for intimate partner violence. Women Health 2012;52:587-605.

17. DeBoer MI, Kothari R, Kothari C, Koestner AL, Rohs T, Jr. What are barriers to nurses screening for intimate partner violence? J Trauma Nurs 2013;20:155-60.; quiz 161-2.

18. Davis RE, Harsh KE. Confronting barriers to universal screening for domestic violence. J Prof Nurs 2001;17:313-20.

19. Waalen J, Goodwin MM, Spitz AM, Petersen R, Saltzman LE. Screening for intimate partner violence by health care providers: barriers and interventions. Amer J Prev Med 2000;19:230-7.

20. Iverson KM, Adjognon OL, Grillo AR, et al. Intimate partner violence screening programs in the Veterans Health Administration: informing scale-up of successful practices. J Gen Intern Med 2019;34(11):2435-42.

21. Hamberger LK, Guse CE, Patel D, Griffin E. Increasing physician inquiry for intimate partner violence in a family medicine setting: placing a screening prompt on the patient record. J Aggression, Maltreatment \& Trauma 2010;19:839-52.

22. Spinola C, Stewart L, Fanslow J, Norton R. Developing and implementing an intervention: evaluation of an emergency department pilot on partner abuse. Eval Health Prof 1998;21:91119. 
23. McColgan MD, Cruz M, McKee J, et al. Results of a multifaceted intimate partner violence training program for pediatric residents. Child Abuse Negl 2010;34:275-83.

24. Wills R, Ritchie M, Wilson M. Improving detection and quality of assessment of child abuse and partner abuse is achievable with a formal organisational change approach. J Paediatr Child Health 2008;44:92-8.

25. Wiist WH, McFarlane J. The effectiveness of an abuse assessment protocol in public health prenatal clinics. Am J Public Health 1999;89:1217-21.

26. Day S, Fox J, Majercik S, Redmond FK, Pugh M, Bledsoe J. Implementing a domestic violence screening program. J Trauma Nurs 2015;22:176-81.

27. Coyer SM, Plonczynski DJ, Baldwin KB, Fox PG. Screening for violence against women in a rural health care clinic. OJRNHC 2006;6:47-54.

28. Decker MR, Frattaroli S, McCaw B, et al. Transforming the healthcare response to intimate partner violence and taking best practices to scale. J Womens Health (Larchmt) 2012;21:1222-9.

29. Danitz SB, Stirman SW, Grillo AR, et al. When usercentered design meets implementation science: integrating provider perspectives in the development of an intimate partner violence intervention for women treated in the United States' largest integrated healthcare system. BMC Womens Health 2019;19:145.

30. Iverson KM. Practical implications of research on intimate partner violence against women. PTSD Research Quarterly 2020;31:1-4.

31. Powell BJ, Waltz TJ, Chinman MJ, et al. A refined compilation of implementation strategies: results from the Expert Recommendations for Implementing Change (ERIC) project. Implement Sci 2015; 10:21.

32. Perry CK, Damschroder LJ, Hemler JR, Woodson TT, Ono SS, Cohen DJ. Specifying and comparing implementation strategies across seven large implementation interventions: a practical application of theory. Implement Sci 2019;14:32.

33. Bazemore A, Neale AV, Lupo P, Seehusen D. advancing the science of implementation in primary health care. J Am Board Fam Med 2018;31:307-11.

34. Gerber MR, Iverson KM, Dichter ME, Klap R, Latta RE. Women veterans and intimate partner violence: current state of knowledge and future directions. J Womens Health (Larchmt) 2014;23: 302-9.

35. Veterans Health Administration Domestic Violence Task Force. Plan for implementation of the Domestic Violence/Intimate Partner Violence Assistance Program. Department of Veterans Affairs, Veterans Health Administration. 2013.

36. Palinkas LA, Horwitz SM, Green CA, Wisdom JP, Duan N, Hoagwood K. Purposeful sampling for qualitative data collection and analysis in mixed method implementation research. Adm Policy Ment Health 2015;42:533-44.

37. Harvey G, Kitson A. PARIHS revisited: from heuristic to integrated framework for the successful implementation of knowledge into practice. Implement Sci 2016;11:33.

38. Hamilton A. Qualitative methods in rapid turnaround health services research. Health Services Research \& Development Cyberseminar, 2013.

39. Iverson KM, Wells SY, Wiltsey-Stirman S, Vaughn R, Gerber MR. VHA primary care providers' perspectives on screening female veterans for intimate partner violence: a preliminary assessment. J Fam Viol 2013;28:823-31.

40. Reid SA, Glasser M. Primary care physicians' recognition of and attitudes toward domestic violence. Acad Med 1997;72:51-3.

41. Department of Veterans Affairs, Veterans Health Administration. Intimate Partner Violence Assistance Program, VHA Directive 1198. January 29, 2019. 